\title{
COVID-19, Rate of Case Factors and Nutritional Characteristics of Patients Dying in Italy and Brazil: A Critical Analyze
}

\author{
Eliza Miranda Ramos ${ }^{1,2}$, Antônio Carlos de Abreu ${ }^{3}$, Sandra Luzinete Félix de Freitas ${ }^{4}$, Matheus Dullius de Lima ${ }^{5}$, \\ Francisco José Mendes dos Reis ${ }^{1}$, Hugo Vieira Ramos ${ }^{6}$, Igor Domingos de Souza ${ }^{1}$, \\ Liliane de Mello Santos Bochenek ${ }^{3}$, Alessandro Carvalho da Fonseca ${ }^{6} \&$ Valter Aragão do Nascimento ${ }^{1}$ \\ ${ }^{1}$ Group of Spectroscopy and Bioinformatics Applied to Biodiversity and Health, School of Medicine, \\ Postgraduation Program in Health and Development in the Midwest Region, Faculty of Medicine, Federal \\ University of Mato Grosso do Sul, Campo Grande, Mato Grosso do Sul, Brazil \\ ${ }^{2}$ Brazil Foundation - CAPES \\ ${ }^{3}$ Postgraduation Program in Health and Development in the Midwest Region, Faculty of Medicine, Federal \\ University of Mato Grosso do Sul, Campo Grande, Mato Grosso do Sul, Brazil \\ ${ }^{4}$ Faculty of Nursing, Federal University of Mato Grosso do Sul, Campo Grande, Mato Grosso do Sul, Brazil \\ ${ }^{5}$ City Hall of Campo Grande, MS, Brazil \\ ${ }^{6}$ Brazilian Hospital Services Company - UFGD \\ Correspondence: Eliza Miranda Ramos, Francisco Serra - 147 - Vila Planalto. Campo Grande, MS. CEP - \\ 79009040. Brazil. Tel: 55-67-999-480-071. E-mail: elizamirandaramos@gmail.com
}

Received: March 31, 2020 Accepted: April 26, 2020 Online Published: May 18, 2020

doi:10.5539/gjhs.v12n7p133 URL: https://doi.org/10.5539/gjhs.v12n7p133

\begin{abstract}
Viruses continued to emerge and bring challenges to the global public health system with emerging viruses with respiratory contagion. Previous studies have shown that the increased incidence of certain viral respiratory infections, including influenza and coronavirus, is associated with low levels of Vitamin D, zinc and iron. Elements such as iron, zinc and Vitamin D influence adaptive immunity by inhibiting the proliferation of B cells with differentiation and secretion of immunoglobulins that will supply the proliferation of $\mathrm{T}$ cells and this will result in a more pro-inflammatory response change.
\end{abstract}

Keywords: Zinc, Vitamin D, iron, viruses, malaria, anemia

\section{Introduction}

In February 2020, severe pneumonia caused by Beta Coronavirus (SARS-COV-2) hit northern Italy called (Pan American Health Organization, 2020) the current outbreak, has 368.338 confirmed cases worldwide, with 16.113 deaths, in 23 March 2020 according to the World Health Organization (WHO) in collaboration with Public Health centers in other countries (Pan American Health Organization, 2020).

Transmission occurs similar to other coronaviruses, human-to-human transmission and, depending on the patient's clinical conditions, causes COVID-19 pneumonia (Chan et al., 2020). And the clinical characteristics of COVID-19 have been slowly reported according to epidemiological indicators with cases of occurrence (Zughaier et al., 2014; Chan et al., 2020). The coronavirus which are encapsulated RNA, with positive sense of single stripe, with zoonoticorigin and belonging to the Coronaviridae family (Zughaier et al., 2014; Chan et al., 2020).

There are numerous questions about the nature of the current COVID-19 pandemic in Italy and the sudden start in Brazil (Chan et al., 2020). When analyzing the discrepancy in cases of severe death between areas in the Chineseprovince where the outbreakbegan, useful data regarding the pathogenicity of the virus can be observed (Pan American Health Organization, 2020; Chan et al., 2020). The cases occur in male men with one or more comorbidities, such as kidney, cardiovascular and cerebrovascular diseases and diabetes or hypertension (Prasad, 2009; Chan et al., 2020). Studies carried out during these COVID-19 pandemic days have demonstrated a direct relationship with comorbidities such as cellular immune deficiency, coagulation, coagulative activation, myocardial injury, liver and kidney damage and correlations with secondary bacterial infections (Chan et al., 2020; Pan American Health Organization, 2020). 
The most serious cases occurred in patients who developed lymphopenia and secondary inflammation, that is, notably, these sequelae are related to pre-existing complications of patients who evolved to death (Chan et al., 2020; Pan American Health Organization, 2020).

However, other patients appear to be able to recover with little or no medical intervention and this epidemiological discrepancy has challenged the Brazilian health system to find an answer (Pan American Health Organization, 2020).

Each region has limited data, it is difficult to make robust statements about populations that may be susceptible to COVID-19 (Chan et al., 2020). But it is worth remembering that the severity of diseases such as SARS and coronavirus corresponds strongly to some underlying conditions of the host, such as age, biological sex,immunological health, vitamin deficits of macro elements and micro elements and previous diseases (Zughaier et al., 2014; Chan et al., 2020).

The evolution of patients inCOVID - 19 for serious purposes, such as death, presented trends similar to the initial patient reports of 2015-nCov (Pan American Health Organization, 2020), in this case, elderly patients over 60 years (Chan et al., 2020), history of previous diseases such as heart or respiratory (Prasad, 2007, 2009), smoking, kidney function, hypertension or chronic diseases such as multiple sclerosis, that is, characteristics included in the 57 lethal cases in Brazil until March 23 (Pan American Health Organization, 2020). Severity increases and causes death in people over 50 years after infection, as the patients underlying health plays a critical role in overall susceptibility to COVID-19 (Chan et al., 2020). The main clinical manifestations of COVID-19 in patients include high fever $\left(\geq 38.0^{\circ} \mathrm{C}\right)$, fatigue, non-productive cough, dyspnea and diarrhea (Pan American Health Organization, 2020). However, the laboratoryexamination of the patients seenincludes normals or reduced leukocyte count, reduced lymphocyte count, especially in severe cases, thrombocytopenia, elevated transaminase, elevated lactate dehydrogenase (LDH), elevated creatine kinase (CK) and elevatedmyoglobin (Chan et al., 2020). In severe cases, the disease progresses rapidly in a few days to difficult-to-treatsepticshock, metabolic acidosis and coagulation dysfunction (Pan American Health Organization, 2020). So, the research question set in this study is: Changes in macroelements and microelements such as iron, zinc and Vitamin D can be used as an indicator to carry out a greater control of the risk group for the infectious process of patients with COVID-19 and, thus, perform a preventive infection control in the body with clinical value? In this short review, we opted for a broad search for works published in the period from 2019 to 2020, involving several well-known bibliographicdatabases, such as PubMed, Lilacs, Medline and Cochrane.

\section{IRON}

The World Health Organization defines iron deficiency as the nutritional disorder with the highest clinical prevalence in the world (Kaiser et al., 2006). Iron is a microelement that plays an important role in human metabolism, such as oxygen transport and storage, release and energy reactions in the electron transport chain, conversion of ribose to dexorribose and can be used as a co-factor of some reactions enzymatic and numerous other essential metabolic reactions in the human body (Castro et al., 2011; Piyush et al., 2016).

The hemoglobin has a greater amount of iron in the human body, and through a distribution in the composition of proteins, enzymes and in the form of deposition (ferritinin hemosiderin) the rest is redistributed (Sim et al., 2010). The most severe iron deficiency occurs as iron deficiency anemia and has as clinical symptoms weakness, pallor, dizziness, irritation, tiredness, shortness of breath and loss of appetite (Castro et al., 2011).

And iron deficiency anemia is associated with impairments in the innate immune response and in the adaptive immune response (Amorin et al., 2012). In the Brazilian territory it has been considered serious in the last two decades and recently the Ministry of Health estimates that it affects about $50 \%$ of children and the elderly as well, as pregnant women and has been considered the greatest nutritional problem in the country (Kaiser et al., 2006; Castro et al., 2011).

The biomolecular mechanism of iron deficit in the body by which it provides susceptibility or resistance to viral infections has not been clarified. Susceptibility to viral infections related to iron deficit has been studied since the 1970s (Cardoso et al., 1992).

Studies have associated iron deficiency anemia with defects in both the adaptive immune response and the innate immune response in the body, causing significant changes in the body's protective functions. Hughes et al (2006) demonstrated that the defect in the adaptive immune response includes a reduction in proliferation, differentiation in the number of $\mathrm{T}$ cells, as well as a reduction in the production of cytokines by these cells (Hughes and Kelly, 2006).

When talking about the innate immune response, it is possible to include a reduction in the phagocytic capacity of 
neutrophils, generally related to low myeloperoxity activity and failures in the activity of Killer cells (NK) (Haussler et al., 2013; Jin, Lee and Kim, 2013; Piyush et al., 2016).

But iron deficiency has an effect on immuno competence and thus acts on susceptibility or resistance to infections, data related to iron deficit with resistance to infection has suggested different hypotheses (Kaiser et al., 2006; Piyush et al., 2016). Iron deficiency can predispose humans to infections, however, other studies suggestprotecting against infectious microorganisms caused by iron deficiency (Kaiser et al., 2006). It is known that iron supplementation has already reduced many problems in the Brazilian population (Kaiser et al., 2006).

Iron supplementation considerably increases hemoglobin levels and decreases the incidence of anemia (Haussler et al., 2013; Ampawong et al., 2015). Iron supplementation decreases the prevalence of infections due to the adverse effects of iron deficiency on the immune system and also decreases the reduction in mortality from diarrhea in anemic children (Adjuik et al., 2004; Castro et al., 2011).

However, a rapid systematic review of iron supplementation was able to establish controversial results (Castro et al., 2011), since, it is possible to verify a mixture of evidence for the incidence, duration and severity of several infectious diseases in association with iron supplementation (Castro et al., 2011; Ampawong et al., 2015). However, iron supplementation gradually decreases the incidence of fever, viral respiratory infection and infectious diarrhea (Cardoso et al., 1992; Hughes and Kelly, 2006). But another study brought a higher prevalence of death to patients with complications related to malaria, pneumonia, sepsis and meningitis (Kaiser et al., 2006; Haussler et al., 2013; Ampawong et al., 2015).

A study with a significant sample, on the other hand, did not find rates of incidence and prevalence of diarrhea or respiratory infections among those who received iron supplementation and those who were not supplemented with iron (Haussler et al., 2013; Amorin et al., 2012).

Currently, COVID-19 has been treated with an initial and effective medication against malaria, in this case, hydroxychloroquine (Chain et al., 2020), which started suddenly in Brazil and has already been tested in France and the United States. Hydroxychloroquine is a drug widely used for antimalarial and autoimmune diseases and has a good profile with efficacy and safety (Cardoso et al., 1992; Castro et al., 2011; Ampawong et al., 2015). Hydroxychloroquine is highly effective in reducing viral replication with an effective concentration and has a favorablepenetration into tissues including the lung (Cardoso et al., 1992; Anstey et al., 2002; Ampawong et al., 2015).

With regard to susceptibility or resistance to malaria, iron supplementation is responsible for the high parasitemia found in individuals from an endemic area (Agbnyega et al., 2000). Recent studies have shown that individuals supplemented with iron are more susceptible to malaria (Adjuik et al., 2004; Agbnyega et al., 2000; Amorin et al., 2012), however, others have shown converging results regarding resistance to malaria provided by iron deficiency (Alves et al., 2007).

In malaria, anemia occurs through hemolysis, which is observed destruction of parasitized and non-parasitized red blood cells (Agbnyega et al., 2000; Hughes and Kelly, 2006). Patients diagnosed with chronic malnutrition and iron deficit have lower hemoglobin and erythrocyte levels than neutrophic patients. In a correlated study of malaria and malnutrition with iron deficit in Colombia it was shown that iron deficit has a much higher proportion (Adjuik et al., 2004; Ampawong et al., 2015).

Therefore, in the current population contaminated by COVID-19 in endemic regions, such as Italy, China and Brazil in a pandemic situation, one of the causes to be taken into account is anemia or iron deficit, because if we correlate the development of the disease with the severity in relation to the worsening of malaria (Adjuik et al., 2004; Haussler et al., 2013), it is possible to observe that anemia with significant iron deficit contributed to the increase of malaria episodes in 51.4\% in Brazil (Alves et al., 2007; Hughes and Kelly, 2006).

COVID-19 is a difficult to treatpathogen, and delay in diagnosis is a worsening factor in coronavirus cases, and generally generates severe complications such as high mortality rates in vulnerable populations, such as hypotension, metabolic acidosis and shock and the like. malaria shows the same signs of worsening and complications (Chan et al., 2020).

According to the $\mathrm{WHO}$, profound respiratory changes, that is, respiratory disorder and pulmonary edema are among the clinical characteristics of worsening malaria and currently COVID-19 which occurs with an incidence of $3 \%$ to $10 \%$ in infections caused by the virus and with lethality close to $70 \%$ (Pan American Health Organization, 2020).

The most serious presentation of COVID-19 is the acute respiratory distress syndrome and is characterized by 
diffuse endothelial injury and increased capillarypermeability and these characteristics occur in the acute phase of malaria in patients with significant iron deficiency (Adjuik et al., 2004; Kaiser et al., 2006; Piyush et al., 2016; Chan et al., 2020).

And what we can see is that endothelial lesions probably have a central role in pulmonary pathology due to the adherence of infected red cells (Piyush et al., 2016), with the release of cytokines and significant leukocyte adhesion (Ampawong et al., 2015; Piyush et al., 2016).

Most of the shocked patients in COVID-19 had low peripheral vascular resistance and increased cardiac output despite the cardiacpump's function being preserveddespite the intense sequestration of infected erythrocytes (Kaiser et al., 2006; Piyush et al., 2016).

In the clinical development of the patient, postural hypotension may be secondary to autonomicdysfunction (Villagra et al., 2007). However, severe hypotensiondevelopsabruptly, usually in the course of pulmonary edema, metabolic acidosis or sepsis (Alves et al., 2007; Prasad, 2009; Ampawong et al., 2015).

In anemic patients, the coagulationcascade is considered a critical component in any infection mainly by COVID-19 and can be correlated to the degree of coagulation with the severity of the infection, since the activation of the coagulationcascade is an immunological mechanism, but it does not occurhemorrhage (Cardoso et al., 1992; Hughes and Kelly, 2006; Kaiser et al., 2006).

It is worth mentioning that tissue hypoxia due to an iron or anemine deficit, presents an increase in muscle activity duringthe probable complications, as there is an increase in the nutritional demand for parasitized erythrocytes, inhibition of glucose oxidation in the mitochondria of erythrocytes caused by thiamine deficit (Ampawong et al., 2015; Amorin et al., 2012) fever and the consequent increase in cytokines (Hughes and Kelly, 2006), by decreasing the clearance of lactate by the liver due to reduced hepatic blood flow (Adjuik et al., 2004), usually the sequestration of infected erythrocytes in capillaries and venules causes damage to the adequate supply of oxygen and thus leads to glycolysis anaerobic (Agbnyega et al., 2000), which causes lactic acidosis with an increased ratio of lactate and pyruvate and with this increase in lactate there is a reduction in the clearance of blood flow from the liver due to a decrease in glycogenesis (Andrade et al., 2010), therefore, the results of the latest studies have shown that it is necessary to surveillance for these vulnerable patients is in 2019-nCoV infection (Pan American Health Organization, 2020, Chain et al., 2020).

\section{ZINC}

Zinc is a structural or functional component of variable metalenzymes and metalproteins and participates in many reactions in cell metabolism, specifically in physiological processes related to immune function and antioxidant defense (Amorin et al., 2012).

The World Health Organization reports that in the last decade eating habits have undergone changes and thus, it has interfered in the bioavailability of zinc obtained in the diet (Baum et al., 2010; Pan American Health Organization, 2020).

It is recognized that there are numerous environmental and cultural factors that have caused zinc deficiency (Amorin et al., 2012), such as low consumption of this element in the mineral diet, energy-protein malnutrition, presence of agents in the diet that decrease its bioavailability, such as fibers and phytates, malabsorption syndrome, chronic liver disease, diabetes mellitus, alcoholism, total parenteral nutrition without zinc supplementation, chelating drugs, for example, penicillamine and also, genetic problems (Haussler et al., 2013).

Recently in Brazil, the nutritional recommendation was modified for the healthy population, in this case $8 \mathrm{mg} /$ day for women and $11 \mathrm{mg} /$ day for men (Haussler et al., 2013; Pan American Health Organization, 2020).

Generally, zinc deficiency has an estimated prevalence of around $45 \%$ of the Brazilian population and $25 \%$ of the world population (Piyush et al., 2016; Pan American Health Organization, 2020), puttingthem at risk, especially in developing countries (Piyush et al., 2016).

Patients with a history of autoimmune diseases, pregnant women, the elderly or individuals on restrictive diets are characterized as risk subgroups due to insufficient low daily zinc intake and tend to develop a subclinical deficiency (Piyush et al., 2016). Among the subclinical signs, impairment of the immune system, changes in taste and smell with significant loss of appetite and dysfunction such as diarrhea can be observed (Zhong et al., 2003).

Zinc deficiency alters the immune system and favors opportunistic viral infections and thus increases mortality rates (Prasad, 2007, 2009).

Otherwise, other studies have shown that supplementing with zinc can improve the immune defense of these 
patients. For example, some studies have reported over the years that zinc deficiency aids in HIV replication and thus impairs cellular immunity and accelerates the apoptosis of cells involved in the immune response (Piyush et al., 2016). For example, $15 \mathrm{mg}$ of chelated zinc for six months was offered to 31 patients who were divided into a zinc group and a placebo group according to plasma zinc levels (Ampawong et al., 2015).

It was possible to report that zinc supplementation significantly increased the $\mathrm{CD} 4+$ cell count in patients with zinc deficiency in relation to the placebo group (Baum et al., 2010). This analysis was carried out in order to verify in the study the effect on the prevention of immune failure among HIV-infected adults (Faw Zi et al., 2005).

In a prospective, double-blind study similar to the previous ones cited by Kaiser et al (2006) to determine the supplementation of micronutrients such as zinc on HIV (Baum et al., 2010), 40 infected patients participated who were supplemented with $30 \mathrm{mg}$ of zinc for twelve weeks at the same time other nutrients such as iron and vitamin D (Faw Zi et al., 2005).

And at the end of the study, the average CD4+ count increased significantly in one of 65 cells in the group supplemented with micronutrients (Baum et al., 2010). Another study showed a positive result, the study by Baum et al (2010) demonstrated that with the significant increase in plasma zinc levels, it is possible to established an average CD4+ cell count (Baum et al., 2010).

When addressing diarrhea in infectious and viral processes such as COVID-19 (Chan et al., 2020), it is possible to observe that zinc supplementation significantly reduces the rate of diarrhea by more than half over time compared to placebo as performed in the study by Baum et al (2010) (Baum et al., 2010; Chan et al., 2020).

Therefore, through the analysis of studies with HIV and if we make a comparison in the development of viral disease (Baum et al., 2010), it is possible to verify the possibility of considering zinc supplementation in patients with COVID-19 important (Baum et al., 2010; Piyush et al., 2016; Chan et al., 2020), however, it should be constantly monitored, since supplements monitored by healthcare professionals in a multidisciplinary way (doctor, nurse and nutritionist) moderately in patients with zinc deficiency can help (Baum et al., 2010).

Each population or health agency in your country should check zinc supplementation strategies for populations at risk, such as preventive conduct (Kaiser et al., 2006), in addition to the importance of standardizing the amounts of zinc to be offered, in order not to exceed the ideal levels of maximum allowed intake so as not to risk the evaluated population (Prasad, 2009; Piyush, 2016).

\section{VITAMIN D}

Currently, in Italy it has been shown that in the last days in Turin that the patient with COVID-19 has shown a high prevalence of Vitamin D deficiency (Mocchegiani et al., 2006; Ramos et al., 2019). In Italy, hypovitaminosis D affects a large part of the inhabitants, especially the elderly, due to highlight that Italy is the second nation with the largest elderly population in the world, after Japan and to be elderly is to be in the risk group of the new COVID-19 (Mocchegiani et al., 2006; Ramos et al., 2019).

Italy recorded the highest number of deaths in the world as a result of COVID-19 at around 4579 cases confirmed and 159 death of March 30 (Pan American Health Organization, 2020). Scientific evidence in previous studies such as tuberculosis, sepsis or flu viruses indicates that the compound has an effect in reducing the risk of infections (Mocchegiani et al., 2006; Ramos et al., 2019), mainly of viral origin, including that of the coronavirus and other studies have shown that Vitamin D is able to fight lung damage caused by inflammation or infection (Prasad, 2009; Ramos et al., 2019).

The role of Vitamin D as an immunomodulators is recognized, since Vitamin D identifies and cancels the action of inflammatory cytokines, mainly interleukins 6 (IL-6) (Haussler et al., 2013; Jin, Lee and Kim, 2013; Ramos et al., 2019), which inducessyndromes of systemic inflammatory response (Ramos et al., 2019). In Brazil children and adults with levels less than or equal to $20 \mathrm{ng} / \mathrm{ml}$ are usually categorized as hypovitaminosis D (Ramos et al., 2019).

However, the low serum concentration of 25-hydroxyvitamin-D2 and 25(OH)D3 are considered an important risk factor forsusceptibility to viral infection and the development of sepsis (Vogeser, 2010; Ramos et al., 2019). For example, low serum levels of Vitamin D-binding protein considered the main Vitamin D transporter decreases in sepsis to levels much below the standard (Jin, Lee and Kim, 2013).

The Vitamin D has a regulatory function for systemic inflammatory cytokine levels such as TNF- $\alpha$ and IL-6 and this effective reaction of Vitamin D is important in the pathogenic development of viral diseases and sepsis (Prasad, 2009).

Infectious diseases tend to show progression that focuses on the dysregulation of inflammatory responses that includes the large and uncontrolled release of pro-inflammatory mediators to initiate a chain of events that lead to 
generalized tissue injuries (Jin, Lee and Kim, 2013; Ramos et al., 2019). Generally, the severity of the immune dysfunction is related to the pathogen process since the cells of the innate or adaptive immune system express the Vitamin D receptor in contrast to the inhibitory role of adaptive immunity (Ramos et al., 2019).

Vitamin D occurs in two forms, in this case, ergocalciferol (Vitamin D2) and cholecalciferol (Vitamin D3) (Jin, Lee and Kim, 2013), both Vitamin D2 and Vitamin D3 have their molecules structured through their inactive molecular formula and activation occurs in the liver and kidney by adding chains to the hydroxyl groups which will result in the predominant active hormonal form, for example, calcitriol (Jin, Lee and Kim, 2013).

The absorption of Vitamin D in the human body occurs by natural food or in the form of supplementation, however it is important to keep Vitamin D in suspension in the proximal small intestine in order to be absorbed (Mocchegiani et al., 2006; Ramos et al., 2019).

Vitamin D is a key regulator of the innate and adaptive immune system and the patient at risk, such as diabetics, heart disease, lung disease, autoimmune, smokers and other chronic or degenerative diseases, has a high prevalence in Vitamin D deficit (Ramos et al., 2019).

An epidemiological study has shown thatVitamin D deficit (blood dose below 20ng/ml) is a risk factor for increased viral infections or development of sepsis in intensive care patients (Vogeser, 2010; Ramos et al., 2019).

Previous studies have indicated that the dosage of vitamin D supplementation in patients in risk groups with a dose equal to or greater than $1250 \mathrm{mcg}(50.000 \mathrm{I} /$ weeks $)$ has no trivial and positive effect on the development of infectious viral diseases, such as H1N1 (Ramos et al., 2019).

A previous meta-analysis study showed that blood serum Vitamin D levels below $20 \mathrm{ng} / \mathrm{ml}$ showed worsening in patients in intensive care with sepsis, mainly in death. Patients who did not routinely monitor vitamin D supplementation $(250 \mathrm{mcg} /$ day $)$ during hospitalization in intensive care showed negative results in improving their clinical condition and generally progressed to death (Ramos et al., 2019).

It is reinforced that this action of Vitamin D to endogenouslyprotect the patient occurs due to the activation mediated by Vitamin D through Toll-like receptors where it occurs or increased resistance to the human catelicidin antimicrobial peptide and thus decreases the vulnerability to infections of viral or bacterial order (Ramos et al., 2019).

In studies with the pediatricpopulation previously, it is possible to verify that vitamin D supplementation increases serum IgG levels with peripheral increase in serum IgM levels, as well as maintaining the high percentage of serum catelicidin (Mocchegiani et al., 2006; Ramos et al., 2019).

Still, in a previous study it is possible to observe a direct relationship between the level of Vitamin $\mathrm{D}$ and mortality in infectious processes such as viruses and bacteria (Vogeser, 2010). The low levels of Vitamin D in the admission of patients in intensive care with worsening by sepsis is directly related to the deficit of Vitamin $\mathrm{D}$ and the time of supplementation. That is, vitamin D supplementation after 12 weeks $(1250 \mathrm{mcg} / \mathrm{week})$ demonstrates an increase in IL-10 production and a decrease in the frequency of Th17 cells in parallel with the expected increases in serum 25(OH)D (Ramos et al., 2019; 2019).

And it is reinforced that Vitamin D was previously associated with anemia in several healthy and sick populations (Tomer et al., 2001), since recent studies indicate that the association may differ between race and ethnic groups and is probably specific for inflammatory or infectious anemia, as for example in sepsis (Ramos et al., 2019).

The mechanism underlying this association involves the reduction of pro-inflammatory cytokines by vitamin $\mathrm{D}$ and the direct suppression of hepcidin mRNA transcription (Mocchegiani et al., 2006; Ramos et al., 2019).

There is also evidence that vitamin $\mathrm{D}$ can be protective against anemia, supporting erythropoiesis. Other calciotropic hormones, including fibroblast growth factor 23 and parathyroid hormone, have also been associated with iron homeostasis and erythropoiesis (Mocchegiani et al., 2006; Ramos et al., 2019).

Even after 12 weeks of Vitamin D supplementation, there is a benefit of Vitamin D in an acute phase protein that is synthesized in the liver in response to cytokines, which aims to reflect systemic active inflammation, in this case, C-reactive protein (PCR) and this process occurs due to the total antioxidant capacity of blood biomarkers of oxidative stress with Vitamin D receptor (Andrade et al., 2010; Ramos et al., 2019).

In short, vitamin D supplementation with a dose equivalent to $1250 \mathrm{mcg} /$ weeks is considered safe and protective against infections in the acute respiratory tract, because Vitamin D works as an endogenous immunomodulatory agent including the regulation of antiviral peptides that are part of innate immunity and can, for example, inactivate the influenza or other viruses (Ramos et al., 2019). 


\section{Conclusion}

Iron, zinc and vitamin D deficiency is a nutritional disorder with the highest clinical frequency in the world. And severe complications in vulnerable populations, for example, diabetics, hypertension, heart disease, anemics and hypovitaminosis D, worsens in cases of viral or bacterial diseases, for example, H1N1 and coronavirus.

\section{Competing Interests Statement}

The authors declare that there are no competing or potential conflicts of interest.

\section{References}

Adjuik M., Babiker A., Garner P., Oliaro P., Taylor W., White N., \& International Artemisinim Study Group. (2004). Artesunate combinations for treatment of malaria. Lancet, 363(9402), e9-17. Artesunate combinations for treatment of malaria: meta-analysis. (2004). https://doi.org/10.1016/S0140-6736(03)15162-8

Agbenyega T., Angus B. J., Bedu-Addo G., Baffoe-Bonnie B., Guyton T., Stacpoole P. W., \& Krishna S. (2000). Glucose and lactate kinetics in children with severe malaria. J Clin Endocrinol Metab., 85(4), 1569-76. https://doi.org/10.1210/jcem.85.4.6529

Alves A., Martins A., Adolphsson S., Bockorny B., Carleti G., Cabral G., Souza A. C. P., \& Vianna A. (2007). Serious imported malaria. Case report. Rev. bras. Intensive therapy, 19(2), São Paulo. https://doi.org/10.1590/S0103-507X2007000200016

Ampawong S., Chaisri U., Viriyavejakul P., Prapansilp P., Georges E. G., Gareth D. H. T., \& Emsri P. (2015). A potential role for interleukin-33 and $\gamma$-epithelium sodium channel in the pathogenesis of human malaria associated lung injury. Malar J, 5(389), 2-15. https://doi.org/10.1186/s12936-015-0922-x

Amorim A. G., \& Tirapegui J. (2012). In: Minerais na Atividade Física: Cálcio, Magnésio, Ferro, Zinco e Cobre. In: TIRAPEGUI J. Nutrição, Metabolismo e Suplementação na Atividade física, 2 ed. São Paulo, Atheneu.

Ananda A. C., Ramji C., Narul A. S., \& Singh W. (1992). C, et al. Malarial hepatitis; a heterogenous syndrome? National Med J, 5(2), 59-62.

Andrade B. B., Reis-Filho A., Barros A. M., Souza-Neto S. M., Nogueira L. L., Fukutani K. F., Camargo E. P., Camargo L. M., Barral A., Duarte A., \& Barral-Neto M. (2010). Towards a precise test for malaria diagnosis in the Brazilian Amazon: comparison among field microscopy, a rapid diagnostic test, nested PCR, and a computational expert system based on artificial neural networks. Malar $J, 6(9), 117$. https://doi.org/10.1186/1475-2875-9-117

Anstey N. M., Jacups S. P., Cain T., Pearson T., Ziesgin P. J., Fisher D. A., Currie B. J., Marks P. J., \& Maguire G. P. (2002). Pulmonary manifestations of uncomplicated falciparum and vivax malaria: cough, small airways obstruction, impaired gas transfer, and increased pulmonary phagocytic activity. J Infect Dis, 185(9), 1326-1334. https://doi.org/10.1086/339885

Baum M. K., Lai S., Sales S., Page J. B., \& Campa A. (2010). Randomized, controlled clinical trial of zinc supplementation to prevent immunological failure in HIV-infected adults. Clin Infect Dis., 50(12), 1653-60. https://doi.org/10.1086/652864

Castro T. G., Silva-Nunes M., Conde W. L., Muniz P.T., \& Cardoso M.A. (2011). Anemia and iron deficiency in preschoolers in the Western Brazilian Amazon: prevalence and associated factors. Cad. Public Health, 27(1), 131-42. https://doi.org/10.1590/S0102-311X2011000100014

Cardoso M. A., Ferreira U. M., Camargo L. M. A., \& Szarfarc S. C. (1992). Anaemia in a population sample from an endemic malaria area of Rondônia State, Brazil. Rev. Saúde Pública, 26(3). https://doi.org/10.1590/S0034-89101992000300006

Chan, J. F., Yuan, S., Kok, K. H., To, K. K., Chu, H., Yang, J., ... \&Yuen, K. Y. (2020). A familial cluster of pneumonia associated with the 2019 novel coronavirus indicating person-to-person transmission: a study of a family cluster. Lancet, Jan 24. PubMed PMID: 31986261. https://doi.org/10.1016/S0140-6736(20)30154-9

Fawzi W. W., Villamor E., Msamanga G. I., Antelman G., Aboud S., Urassa W., \& Hunter D. (2005). Trial of zinc supplements in relation to pregnancy outcomes, hematologic indicators, and T cell counts among HIV-1 infected women in Tanzania. Am J Clin Nutr., 81(1), 161-7. https://doi.org/10.1093/ajcn/81.1.161

Hughes, S., \& Kelly, P. (2006). Interactions of malnutrition and im-16. imune impairment, with specific reference to immunity against parasites. Parasite Immunol., 28, 577-88. 
https://doi.org/10.1111/j.1365-3024.2006.00897.x

Haussler, M. R., Whitfield, G. K., Kaneko, I., Haussler, C. A., Hsieh, D., \& Hsieh, J. C. (2013). Molecular mechanisms of vitamin D action. Calcif Tissue Int., 92, 77-98. https://doi.org/10.1007/s00223-012-9619-0

Iannotti, L. L., Tielsch, J. M., Black, M. M., \& Black, R. E. (2006). Iron sup-21. Supplementation in early childhood: health benefits and risks. Am J Clin Nutr., 84, 1261-76. https://doi.org/10.1093/ajcn/84.6.1261

Jin, H. J., Lee, J. H., \& Kim, M. K. (2013). The prevalence of vitamin D deficiency in iron-deficient and normal children under the age of 24 months. Blood Res., 48, 40-45. https://doi.org/10.5045/br.2013.48.1.40

Kaiser, J. D., Campa, A. M., Ondercin, J. P., Leoung, G. S., Pless, R. F., \& Baum, M. K. (2006). Micronutrient Supplementation Increases CD4 Count in HIV-Infected Individuals on Highly Active Antiretroviral Therapy: A Prospective, Double-Blinded, Placebo-Controlled Trial. J Acquir Immune Defic Syndr., 42(5), 523-8. https://doi.org/10.1097/01.qai.0000230529.25083.42

Mocchegiani, E., Costarelli, L., Giacconi, R., Cipriano, C., Muti, E., Tesei, S., \& Malavolta, M. (2006). Nutrient-gene interaction in ageing and success ful ageing: a single nutrient (zinc) and some target genes related to inflammatory/immune response. Mechanisms of ageing and development, 127(6), 517-525. https://doi.org/10.1016/j.mad.2006.01.010

Piyush, G., Pooja, D., Dheera, J. S., Nisha, S., Nidhi, B., Iqbal, R. K., Ajay, K. B., \& Sv, M. (2016). Vitamin D Supplementation for Treatment and Prevention of Pneumonia in Under-five Children: A Randomized Double-blind Placebo Controlled Trial. INDIAN PEDIATRICS, 53. https://doi.org/10.1007/s13312-016-0970-5

Prasad A. S. (2007). Zinc: mechanisms of host defense. The Journal of nutrition. [s.l.], 137(5), 1345-1349. https://doi.org/10.1093/jn/137.5.1345

Prasad. A. S. (2009). Zinc: role in immunity, oxidative stress and chronic inflammation. Current Opinion in Clinical Nutrition \& Metabolic Care. [s.l.], 12(6), 646-652. https://doi.org/10.1097/MCO.0b013e3283312956

Pan American Health Organization, OPAS. Folha informativa - COVID-19 (doença causada pelo novo coronavírus)

(2020).

From https://www.paho.org/bra/index.php?option=com_content\&view=article\&id=6101:covid19\&Itemid=875.

Ramos, E. M., Lima, M. D., Gonzalez, J. E. C., Facco, G. G., \& Nascimento, D. V. A. (2019). Vitamin d in intensive therapy in treatment of adult patients: a methanalysis of clinical testing. International Journal of Development Research, 9.

Ramos, E. M., Lima, M. D., Gonzalez, J. E. C., Facco, G. G., \& Nascimento, D. V. A. (2019). Analysis of vitamin $\mathrm{d}$ effectiveness of short interventions in the prevention and reduction of sepsis in Brazilian intensive care: a systematic review. International Journal of Development Research, 9(2).

Snow, R. W., Byass, P., Shenton, F. C., \& Greenwood, B. M. (1991). The relationship between anthropometric measurements and measurements of iron status and susceptibility to malaria in gambia children. Transactions of the Royal Society of Tropical Medicine and Hygiene, London, 85(5), 584-589. https://doi.org/10.1016/0035-9203(91)90351-X

Sim J. J., Lac P. T., Liu I. L., Merguerditchian S. O., Kumar V. A., Kujubu D. A., \& Rasgon S. A. (2010). Vitamin D deficiency and anemia: a cross-sectional study. Ann Hematol, 89, 447-452. https://doi.org/10.1007/s00277-009-0850-3

Tomer, A., Harker, L. A., Kasey, S., \& Eckman, J. R. (2001). Thrombogenesis in sickle cell disease. J Lab Clin Med., 137(6), 398-407. https://doi.org/10.1067/mlc.2001.115450

Vogeser, M. (2010). Quantification of circulating 25-hydroxyvitamin D by liquid chromatography - tandem mass spectrometry. J Steroid Biochem Mol Biol., 121(16-17), 565-73. https://doi.org/10.1016/j.jsbmb.2010.02.025

Villagra, J., Shiva, S., Hunter, L. A., Machado, R. F., Gladwin, M. T., \& Kato, G. J. (2007). Platelet activation in patients with sickle disease, hemolysis associated pulmonary hypertension, and nitric oxide scavenging by cell-free hemoglobin. Blood, 110(6), 2166-72. https://doi.org/10.1182/blood-2006-12-061697

Zughaier, S. M., Alvarez, J. A., Sloan, J. H., Konrad, H. R., \&Tangpricha, V. (2014). The role of vitamin D in the regulation of the iron-hepcidinferroportin axis in monocytes. Journal of Clinical and TranslationalEndocrinology, 1(01), e19-e25. https://doi.org/10.1182/blood-2006-12-061697 
Zhong, N. S., Zheng, B. J., Li, Y. M., Poon, Xie, Z. H., Chan, K. H., Li, P. H., ... \& Guan Y. (2003). Epidemiology and cause of severe acute respiratory syndrome (SARS) in Guangdong, People's Republic of China, in February, 2003. Lancet, 25(362), (9393), 1353-8. https://doi.org/10.1177/095632020501600102

\section{Copyrights}

Copyright for this article is retained by the author(s), with first publication rights granted to the journal.

This is an open-access article distributed under the terms and conditions of the Creative Commons Attribution license (http://creativecommons.org/licenses/by/4.0/). 